\title{
Infections in acute leukemia in Indian Children
}

\author{
B Roy, ${ }^{1}$ A Biswas, ${ }^{2}$ A Chaterjee, ${ }^{3}$ A Pan ${ }^{4}, \mathrm{~K} \mathrm{Basu}^{5}$ \\ ${ }^{1}$ Associate Professor, NRS Medical College, Kolkata, ${ }^{2}$ RMO Cum Clinical Tutor,IPGME \&R and SSKM \\ Hospital,Kolkata, ${ }^{3}$ Assistant Professor, National Medical College,Kolkata, ${ }^{4}$ Lecturer,College of Medical \\ Sciences, Bharatpur, ${ }^{5}$ Professor,Department of Pediatrics, Medical College Kolkata,India.
}

\section{Abstract \\ Aims}

In the present study acute leukemic children were studied to determine the incidence and principal site of infection, correlation with absolute neutrophil count, causative organisms and to standardize the initial empirical anti microbial therapy.

\section{Materials and methods}

A total 40 children in the age group 6 month to 12 year with acute leukemia relapse were included in this study. A total 82 infectious episodes including 61 febrile episodes were investigated for infectious etiology.

\section{Results}

We found that the frequency of infections increased significantly with the degree of immunocompromisation specially neutropenia (ANC $<500 / \mathrm{cmm}$ ). The skin and soft tissue was the commonest site of infection (26.83\%), followed by respiratory tract (21.95\%). Staphylococcus nonhemolytic coagulase-negative (34\%), followed by Klebsiella (17\%) were the most common organisms isolated from blood. Staphylococcus non-hemolytic coagulase-negative was also the commonest isolate (26\%) from other sites of infection. Most strains were sensitive to Cloxacillin, cephalosporin and aminoglycosides.

\section{Conclusion}

For the treatment of febrile episodes, empirical use of beta-lactamase resistant penicillin e.g. Cloxacillin or cephalosporin combined with an aminoglycosides with a broad spectrum antifungal like fluconazole in selective cases at the first sign of infection is recommended.

\section{Key words: Infection, acute leukemia, children, antimicrobial therapy.}

\section{Introduction}

Patients with hematological malignancies such as leukemia are susceptible to infectious complications. The host is immunocompromised by both malignancy and cytotoxic treatment. As a result, infections become an important cause of morbidity and mortality. With

Correspondence: Dr. Arnab Biswas

E-mail :dr24arnab@rediffmail.com the judicious use of antibiotics and supportive treatment, the rate and duration of remission and survival have improved greatly. To optimize survival, the pattern of infection must be determined in these patients so that effective empiric therapy must be instituted at the earliest sign of infection. ${ }^{1}$ 


\section{Aims}

This study aims to:

1. Determine the incidence of infection in relation to the severity of immunocompromisation specially the absolute neutrophil count.

2. Determine the common site of infection of these immunocompromised patients.

3. Identify the pathogens isolated in febrile patients with acute leukemia and their sensitivity pattern to common antibiotic regimen.

\section{Materials and methods}

The study was conducted at inpatient pediatric ward, medical college Kolkata. A total number of 40 children, suffering from acute leukemia, in the age group upto 12 year, admitted during the period January 2007 to February 2008, formed the study group.

Diagnosis of leukemia was based on blood and bone marrow examinations. All patients were put on standard chemotherapy.

Complete blood count with actual platelet count, blood cultures, chest $\mathrm{X}$-rays and urinalysis were done in all patients. Cultures from other sites such as wound discharge, ear discharge, throat swab, stool, and vaginal discharge were done in selected cases. Viral studies were not done because of financial constraints. A significant febrile episode was defined as three oral temperature elevations above $38^{\circ} \mathrm{C}$ over a period of 24 hour or a single temperature elevation above $38.5^{\circ} \mathrm{C}$, which could not be attributed to any blood transfusion, chemotherapy or allergic reactions etc.

The diagnosis of pneumonia was considered if the patient had a compatible chest $\mathrm{x}$-ray examination and clinical presentation. Often the etiologic agent could not be identified because gram staining and culture of the sputum were not done.

Septicemia was defined as the presence of at least one positive blood culture in a patient.

Gastrointestinal tract infections included oral infections such as thrush or significant ulcerations containing pathogenic organisms, positive throat swab culture, dental infections and enteritis.

Genito-urinary tract infections included all those patients with urinary tract infections and vulvar abscess. Urinary tract infection was considered to be present if the patient had clinical presentation and with pus cells of greater than 10 cells in urinalysis or greater than or equal to $100,00 \mathrm{col} / \mathrm{ml}$ of a single organism cultured from the urine.

The presence of cellulitis was established based on clinical signs.

The patient was said to be in remission if there was freedom from symptoms and physical findings attributable to leukemia, normal peripheral blood cell values, $1,000 / \mathrm{mm}$. or more neutrophils and $150,000 /$ $\mathrm{mm}$. or adequate platelets, hemoglobin of 10 grams or more, no demonstrable blast and bone marrow blast less than 5\% with megakaryocytic, granulocytic and erythroid activity intact.

Patient was on relapse when there was a reappearance of leukemia in the blood, bone marrow, skin, CNS, testes or other visceral organs. 
Patient was on induction therapy when the initial chemotherapy led to a complete remission usually within4-8weeks.

On suspicion of infection, all patients were started on a combination of aminoglycosides and Cephalosporins or beta-lactamase resistant penicillin given intravenously. Antibiotics were adjusted according to the results of culture and sensitivity test and the therapeutic response. No post-mortem examination was done in any of these patients.

\section{Results}

There were 47 hospitalizations for a total of 40 patients of which 34 children (85\%) had acute lymphoblastic leukemia (ALL) and 24 had acute myelogenous leukemia (AML). Four patients were readmitted because of repeated infections from different foci. Twenty-eight children were male and 12 were female, with a male to female ratio of $2.3: 1$.
A total of 61 febrile episodes were evaluated for infective etiology. Organisms were isolated in $40 \%$ cases at presentation and $92 \%$ cases at induction. This finding is statistically significant $(\mathrm{p}<0.01)$. (Table. 1$)$

Table 1: Time of infectious episodes

\begin{tabular}{|c|c|c|}
\hline $\begin{array}{l}\text { Time of febrile } \\
\text { episode } \\
\text { No }(\%)\end{array}$ & $\begin{array}{c}\text { Infection not } \\
\text { identified } \\
\text { No }(\%)\end{array}$ & $\begin{array}{c}\text { Infection } \\
\text { identified } \\
\text { No }(\%)\end{array}$ \\
\hline \multicolumn{3}{|l|}{ At presentation } \\
\hline $30(49.18 \%)$ & $18(60 \%)$ & $12(40 \%)$ \\
\hline \multicolumn{3}{|l|}{ During induction } \\
\hline $25(40.99 \%)$ & $2(8 \%)$ & $23(92 \%)$ \\
\hline \multicolumn{3}{|l|}{ During remission } \\
\hline $6(9.84 \%)$ & $4(66.66 \%)$ & $2(33.33 \%)$ \\
\hline Total 61 & $24(39.34 \%)$ & $37(60.66 \%)$ \\
\hline
\end{tabular}

Organisms were isolated more frequently $(81.25 \%)$ if febrile episode occurred in patients with Absolute Neutrophil Count (ANC) $<500 / \mathrm{cmm}$ and a much less chance of isolation of organisms (48.48\%) when ANC was $>1500$. (Table. 2 )

Table 2: Relation with ANC

\begin{tabular}{lccc}
\hline $\begin{array}{l}\text { Absolute neutrophilcount } \\
\text { [ANC](cells/cmm) }\end{array}$ & $\begin{array}{c}\text { Febrile } \\
\text { episodes }\end{array}$ & $\begin{array}{c}\text { Infection } \\
\text { identifiedNo (\%) }\end{array}$ & $\begin{array}{c}\text { Infection not } \\
\text { identified No (\%) }\end{array}$ \\
\hline$<500$ & 16 & $13(81.25 \%)$ & $3(18.75 \%)$ \\
$500-1500 *$ & 12 & $8(66.66 \%)$ & $4(33.33 \%)$ \\
$>1500 * *$ & 33 & $16(48.48 \%)$ & $17(51.51 \%)$ \\
\hline Total & $\mathbf{6 1}$ & $\mathbf{3 7}(\mathbf{6 0 . 6 6 \% )}$ & $\mathbf{2 4 ( 3 9 . 3 4 \% )}$ \\
\hline$*<0.01$ & $* * 0.02$ & &
\end{tabular}


B Roy et al, Infection in acute leukemia in Indian

Table. 3 shows that the skin and soft tissue was the commonest site of infection, accounting for $26.83 \%$ of all infection, closely followed by respiratory tract (21.95\%).

Table 3 Site of infection in acute leukemia patients

\begin{tabular}{|c|c|c|c|c|}
\hline Sites of infection & $\begin{array}{l}\text { Absolute } \\
<500 / \mathrm{cmm}\end{array}$ & $\begin{array}{c}\text { neutrophil } \\
500-1500 / \mathrm{cmm}\end{array}$ & $\begin{array}{c}\text { count } \\
>1500 / \mathrm{cmm}\end{array}$ & $\begin{array}{c}\text { Total } \\
\text { No }(\%)\end{array}$ \\
\hline Skin and soft tissue & 10 & 7 & 5 & $22(26.83)$ \\
\hline Respiratory tract & 10 & 4 & 4 & $18(21.95)$ \\
\hline Gastrointestinal including oropharynx & 8 & 5 & 3 & $16(19.51)$ \\
\hline Septicemia & 4 & 4 & 2 & $10(12.19)$ \\
\hline Genitourinary & 3 & 2 & 1 & $6(7.32)$ \\
\hline Others & 5 & 2 & 3 & $10(12.19)$ \\
\hline Total & $40(48.78)$ & $24(29.26)$ & $18(21.95)$ & $82(100)$ \\
\hline
\end{tabular}

Most of the infection (48.78\%) occured when the granulocytopenia was severe $(<500 / \mathrm{cmm})$, whereas the incidence of infection reduced considerably $(21.95 \%)$ when the ANC was more than $1500 / \mathrm{cmm}$. Sixty seven percent of pathogens isolated were from the blood (Tables 4 and 4A). Gram-positive organisms were responsible for the $50 \%$ of the 30 episodes in which organisms were identified in blood and $53 \%$ of 15 episodes from other sites.

Staphylococcus non-hemolytic coagulase-negative was the most common isolate, accounting for $34 \%$ in blood and $26 \%$ from other sites (Tables 4 and $4 \mathrm{~A}$ ).

Gram-negative bacilli were responsible for $42.30 \%$ of all the infection including blood and other sites. E.coliand Klebsiella were the gram negative bacilli cultured most frequently from blood and different sites of infection.

In only one episode multiple organisms was cultured from blood (Tables 4).
Table 4: Blood culture results in acute leukemia

\begin{tabular}{ll}
\hline Organisms & Number \\
\hline
\end{tabular}

\section{Gram Positive Pathogens}

Staphylococcus non-hemolytic

coagulase-negative

Staphylococcus non-hemolytic

coagulase-positive

Bacillus subtilis

\section{Gram Negative Pathogens}

Klebsiella

Escherichia coli

Enterobacter

Pseudomonas sp

\section{Fungal}

Candida species

\section{Mixed Pathogens}

Pseudomonas sp. and Escherichia coli

Total 30 
Table 4A: Culture results in acute leukemia from other sites

\begin{tabular}{lcc}
\hline Organisms & Sites & Number \\
\hline Gram Positive Pathogens & & 4 \\
Staphylococcus (non-hemolytic coagulase-positive) & Wound discharge & 1 \\
Staphylococcus (non-hemolytic coagulase-negative) & Wound discharge & 1 \\
Alpha-hemolytic Streptococcus & Throat swab & 2 \\
Bacillus subtilis & Wound discharge \\
Gram Negative Pathogens & Stool & 1 \\
Escherichia coli & Urine & 2 \\
& Ear & 1 \\
Pseudomonas sp & Urine & 1 \\
Klebsiella species & Wound discharge & 2 \\
\hline Total & & $\mathbf{1 5}$ \\
\hline
\end{tabular}

Table 5 shows that Staphylococcus coagulase-negative or positive has acceptable sensitivity to Cloxacillin and Amikacin (72-75\%) and suitable for empiric regimen, whereas vancomycin and linezolide having excellent $(100 \%)$, is a good option for resistant Staphylococcus infection. Third generation cefalosporins (cefepime, ceftazidime) have pretty well efficacy (75-100\%) against gram negative organisms. Levofloxacin has got excellent and wide sensitivity against both gram positive and gram negative organism and therefore a candidate for future empirical therapy.

Table 5: Drug sensitivity pattern of important organisms

\begin{tabular}{|c|c|c|c|c|c|c|c|c|}
\hline Organisms & Cloxacillin & Amikacin & Netilmycin & Cefepime & Ceftazidime & Vancomycin & Linezolid & Levoflxacin \\
\hline \multicolumn{9}{|l|}{$\begin{array}{l}\text { Staphylococcus } \\
\text { (non-hemolytic } \\
\text { coagulase- }\end{array}$} \\
\hline (n=11) & (72.77 \%) & $(72.77 \%)$ & $(90.90 \%)$ & (63.63\%) & (63.63\%) & (90.90\%) & $(100 \%)$ & (72.77 \%) \\
\hline \multicolumn{9}{|l|}{$\begin{array}{l}\text { Staphylococcus } \\
\text { (non-hemolytic }\end{array}$} \\
\hline coagulase-positive) & 6 & 6 & 7 & 6 & 5 & 8 & 8 & 7 \\
\hline$(n=8)$ & $(75 \%)$ & $(75 \%)$ & $(87.50 \%)$ & $(75 \%)$ & $(63.50 \%)$ & $(100 \%)$ & $(100 \%)$ & $(87.50 \%)$ \\
\hline Klebsiella species & & 4 & & 6 & 6 & & & 8 \\
\hline$(n=8)$ & - & $(50 \%)$ & - & $(75 \%)$ & $(75 \%)$ & - & - & $(100 \%)$ \\
\hline Escherichia coli & 1 & 5 & & 5 & 5 & & & 6 \\
\hline$(n=6)$ & $(16.66 \%)$ & $(83.33 \%)$ & - & $(83.33 \%)$ & $(83.33 \%)$ & - & - & $(100 \%)$ \\
\hline Pseudomonas & & 1 & & 1 & 3 & & & 2 \\
\hline$s p(n=3)$ & - & $(33.33 \%)$ & - & $(33.33 \%)$ & $(100 \%)$ & - & - & $(66.66 \%)$ \\
\hline
\end{tabular}




\section{Discussion}

Infection in the immunocompromised host is an important problem in present day medicine. Acute leukemia has a high frequency of infectious complications further influenced by therapy. ${ }^{1-13}$ Infection not only influences the outcome of chemotherapeutic response but also the morbidity of these patients. Thus, prevention and treatment of infection are vital in the management of acute leukemia. Neutropenia is probably the most important factor predisposing to infection in cancer patients. In patients with neutrophil count of 500/cu mm, both the incidence and the rate of recurrence of infection increase significantly. ${ }^{3}$ Granulocytopenia usually occurs in acute leukemia either because of leukemic process or a result of intensive chemotherapy.

Pickering LK has shown in his study that chemotaxis, phagocytosis and intracellular bactericidal activity are markedly reduced. ${ }^{4}$ In our study, the episode of infection was more frequent in those with an ANC of $<500 /$ cu mm. Remission significantly influenced the occurrence of infection. In our study, the incidence of infection in patients on complete remission was markedly lower than in those with induction or relapse. The infection rate in patients on induction is still lower than that of patients on relapse. The result of this study indicates that the decrease in infectious episode was associated with an improved ANC. This decrease may also be associated with the recovery of both the quantity and the function of the granulocytes. ${ }^{4}$

In addition to the deficiencies created by the malignant process itself, many patients acquire deficiencies in host defense mechanism as a consequence of their therapy.
Corticosteroids are administered as part of the chemotherapeutic regimen in acute leukemia. In a study by Qi-nan et al in 1989, they had observed that the incidence of infection and mortality were greater among those on corticosteroids than those not receiving corticosteroids. ${ }^{5}$ This agent has a profound effect on host defense mechanisms, including decreasing antibody production, suppressing acute and chronic inflammatory response reducing delayed hypersensitivity reactions and impairing wound healing. ${ }^{6}$ Since corticosteroids are often a component of chemotherapeutic regimen, strict vigilance should be exercised in monitoring such patients.

The distribution of infections according to site in this study is very different from other investigators. ${ }^{3,7,8} \mathrm{In}$ our study skin and soft tissue was the commonest site of infection, closely followed by respiratory tract, whereas the workers like Sickes EA et al. find that respiratory tract is most commonly affected in these patients. ${ }^{7}$ Application of various invasive procedures frequently and failure to maintain local asepsis may be responsible for this. Contrary to a report by Bodey GP, multiple organisms were uncommon in our patients. $^{9}$

The spectrum of organisms is constantly changing due to modification in chemotherapy. With the incorporation of semi-synthetic anti-staphylococcal penicillins into routine antibiotic regimens, the frequency of fatal S. aureus infections decreased. However, in a recent report by Elihu A, Gollin G, this organism has re-emerged as an important pathogen because of widespread use of intravenous catheters. ${ }^{10}$ The poor 
anti-staphylococcal coverage of most routine antibiotic regimens also contributes. ${ }^{11}$

E. coli and Klebsiella were the most common cause of gram-negative bacillary infections. Several studies including the one by Wang SM et al, have indicated that fungal infections are increasing in frequency, not only in leukemic but in other patients with compromised host defense mechanisms. ${ }^{12}$ However, fungal, organisms were responsible for only a small portion of the total infections in these study. The most frequent fungal infection was oral candidiasis. This was known to develop in patients with cellular immunodeficiencies and in patients treated with cancer chemotherapeutic agents or steroids.

Viral infections were diagnosed infrequently in this study. There were three episodes of measles and one episode of herpes zoster infection.

\section{Conclusion}

This study clearly indicated that the incidence of infection is inversely proportional to the absolute neutrophil count. Infection in acute leukemia was more prevalent before and during the induction phase of chemotherapy. The skin and soft tissue was the commonest site of infection, thereby reflecting the need for more stringent aseptic precaution and cautious attitude of health care providers. The Gram Positive Pathogens esp. Staphylococcus non-hemolytic coagulase-negative, followed by Gram Negative Pathogens including E. coli and Klebsiella were the most common organisms isolated. They remain sensitive to beta-lactam, cephalosporins and aminoglycosides.

\section{Recommendation}

Strict asepsis during any invasive procedure, careful attitude during cytotoxic drug delivery, so that no extravasation occurs, and effective local wound care in time is strongly recommended in order to reduce the incidence of soft tissue infection. It is recommended that a combination of beta-lactam and aminoglycosides be instituted as an empiric drug therapy for infections in acute leukemia. However, better diagnostic technique is required if fungal and viral infections are considered. Newer broad spectrum antibiotics have a definite advantage in terms of efficacy. However, emergence of antibiotic resistance and superinfection needs careful consideration.

These matters will aid in preventing infection or improving the outcome of infection.

\section{References}

1. Advani SH, Banavali SD. Pattern of infection in hematologic malignancies: An Indian experience. Rev Infect Dis 1989;1621-28.

2. Urrea M, Rives S, Cruz O, Navarro A, García JJ, Estella J. Nosocomial infections among pediatric hematology/oncology patients: results of a prospective incidence study. Am J Infect Control. 2004;32(4):205-8.

3. Miane J, Cheesels JM. Serious infections during continuing treatment in acute lymphoblastic leukemia. Arch Dis Child 1981; 56:841-4.

4. Pickering LK. Effect of chemotherapy agents on metabolic and bactericidal activity of polymorphonuclear leucocyte. Cancer 1978; 42:1741-6. 
B Roy et al, Infection in acute leukemia in Indian

5. Qinan, Zhong-Da. Infection in acute leukemia: An analysis of 433 episedes. Rev lnfect Dis 1989; 2(Suppl 7 0:1613.20.

6. Dale DS. Corticosteroids and infectious diseases. Med Clin North Am 1973; 57:1277-87.

7. Sickes EA, Yound VM, Green WH, Wiernik. Pneumonia in acute leukemia. Ann Intern Med 1973; 79:528-34.

8. Lee BW, Yip WCL, Tay JSH, Yap HK, Wong HB, Quah TC, Han P. Severe infections in acute leukemia:J Singapore Pediatr Soc 1988;30:51-5.

9. Bodey GP. Multiple organisms septicemia in acute leukemia. Arch Intern Med 1965;116:266-72.
10. Elihu A, Gollin G. Complications of implanted central venous catheters in neutropenic children.Am Surg. 2007;73(10):1079-82

11. Rubin M, et al. Gram-positive infections and the use of vancomycin in 550 episodes of fever and neutroponia. Ann Intern Med 1988; 108:30-35.

12. Wang SM, Yang YJ, Chen JS, Lin HC, Chi CY, Liu CC. Invasive fungal infections in pediatric patients with leukemia: emphasis on pulmonary and dermatological manifestations. Acta Paediatr Taiwan. 2005;46(3):149-55.

13. Viscoli C. Antibacterial prophylaxis in neutropenic patients. Int J Antimicrob Agents. 2007;30 Suppl 1:S60-5. 